\title{
High energy, single-mode, all-solid-state and tunable UV laser transmitter
}

Narasimha S. Prasad ${ }^{a}$, Upendra N. Singh ${ }^{a}$, Floyd Hovis ${ }^{b}$

aNASA Langley Research Center,

5 N. Dryden St., MS 468, Hampton, VA 23681

bFibertek, Inc.,

510 Herndon Parkway, Herndon, VA 20170

\section{CLEO/Europe-IQEC Conference}

$$
\text { June 17-22, } 2007
$$

World of Photonics Congress 2007 in the International

Congress Centre Munich, Germany

Paper Session Code: CA5-4-TUE 15:30 


\section{Laser Risk Reduction Program (LRRP)}

- NASA began Laser Risk Reduction Program (LRRP) in 2002 to develop reliable, robust, and compact laser technologies for lidar applications from space based platforms

- Program: $\quad$ Joint operation of Langley Research Center and Goddard Space Flight Center

- Goal:

1 micron and 2 micron lasers and wavelength conversion technology

- Applications: Four Lidar Techniques-altimetry, Doppler, Differenctial Absorption Lidar (DIAL), backscatter lidar

- Measurements: 6 priority Earth Science measurements:

(1) Surface and ice mapping, (b) Horizontal vector wind profiles (3) Carbon-di-oxide $\left(\mathrm{CO}_{2}\right)$ profiles (4) Ozone $\left(\mathrm{O}_{3}\right)$ profiles(5) Aerosol/clouds and (6) River currents 


\section{UV Task Objectives}

- The objective of the UV Task is to develop an efficient, all-solid-state, diode pumped, conductively cooled, single longitudinal mode and high energy 1micron to UV wavelength conversion technology

- The emphasis is to generate $U V$ wavelengths of $308 \mathrm{~nm}$ and $320 \mathrm{~nm}$ for ozone sensing using DIfferential Absorption Lidar (DIAL) technique from space

- Performance Goals:

- Output energy at UV wavelengths: $\quad \geq 200 \mathrm{~mJ}$

- Pulsewidth: $10-25$ ns

- PRF: $\quad 50 \mathbf{~ H z}$

- High pulse energy allows enhanced performance during strong daylight conditions

- UV Task is a collaborative effort among Sandia National Labs, Fibertek, and NASA LaRC 


\section{Technical Approach to UV generation}

- Basic Scheme comprises of a Nd:YAG laser pumped nonlinear optics based converter comprising of a second harmonic generation (SHG), optical parametric oscillator, (OPO) and sum frequency generation (SFG) processes
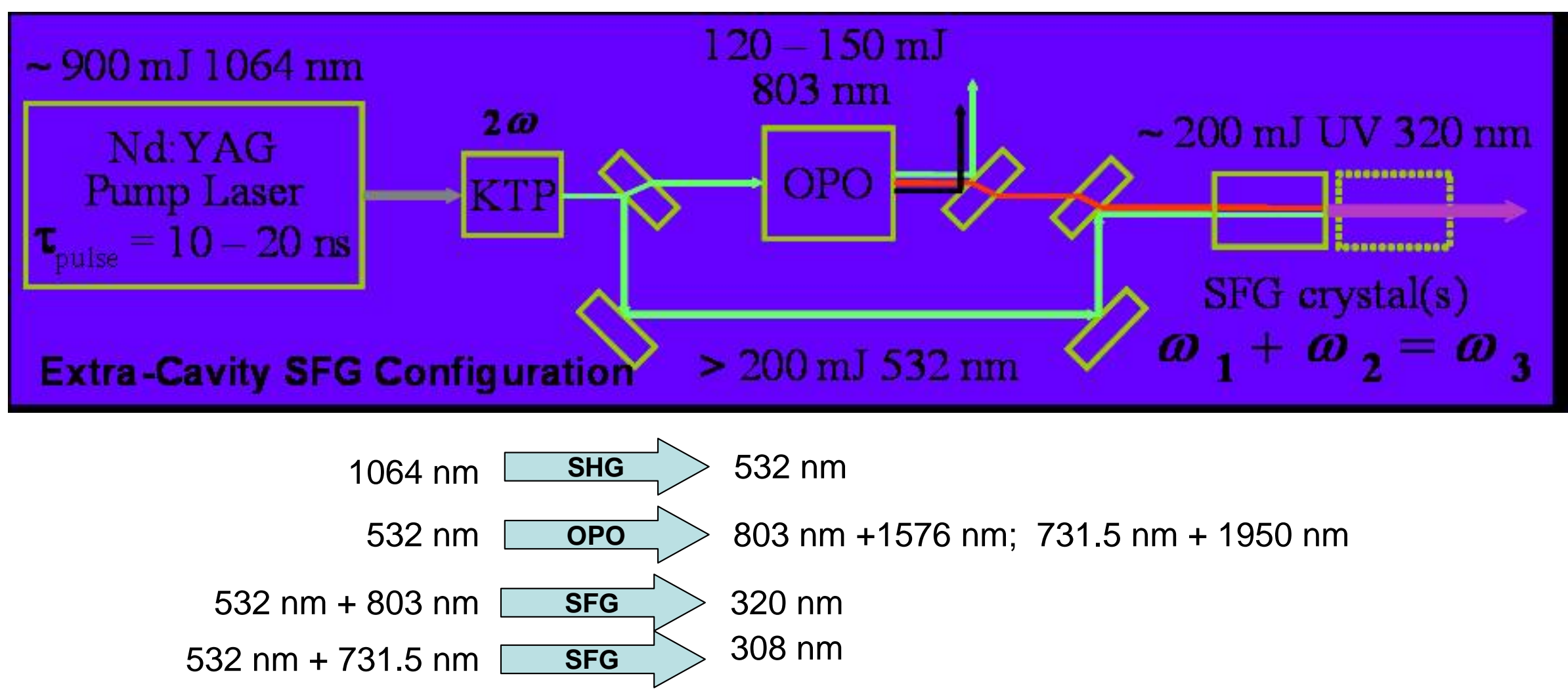


\section{UV Wavelength Conversion -Experimental Results-}

- The nonlinear optics based technology to efficiently generate UV wavelengths has been established using a flash lamp pumped Nd:YAG laser

- The scheme utilizes a novel (Rotated Image Singly Resonant Twisted RectAngle) RISTRA OPO to generate 803 and $731.5 \mathrm{~nm}$ wavelengths pumped using a $532 \mathrm{~nm}$ pump source

- A type-I BBO crystal is used in the RISTRA OPO and a LBO crystal is used for SFG

- Single mode operation is obtained through pulsed seeding technique with temporally matched pump and idler pulse profile

- Pulse idler seeding is obtained by a tunable laser diode and RISTRA OPO in tandem as seed sources

- For 803 nm

- A small or low energy RISTRA OPO that is locked by Pound-Drever-Hall (PDH) technique and seeded by New Focus tunable diode laser operating at $803 \mathrm{~nm}$

- The 1.5x scaled big RISTRA OPO that is pulse seeded at $1576 \mathrm{~nm}$ from the small OPO and locked by energy stabilization technique 


\section{Latest Results on the UV conversion}

- State-of-the-art conversion efficiencies have been demonstrated using a flash lamp pumped Nd:YAG laser with a round top-hat profile

$>$ Greater than $90 \%$ pump depletion obtained

$>$ At $320 \mathrm{~nm},>200 \mathrm{~mJ}$ extra cavity SFG with good beam Quality

- IR to UV efficiency $>21 \%$ ( $27 \%$ for $1 \mathrm{~mJ}$ seed)

$>$ At $320 \mathrm{~nm}$, up to $160 \mathrm{~mJ}$ intra-cavity SFG

- IR to UV efficiency up to $24 \%$

$>$ Fluence $\geq 1 \mathrm{~J} / \mathrm{cm}^{2}$ for most beams

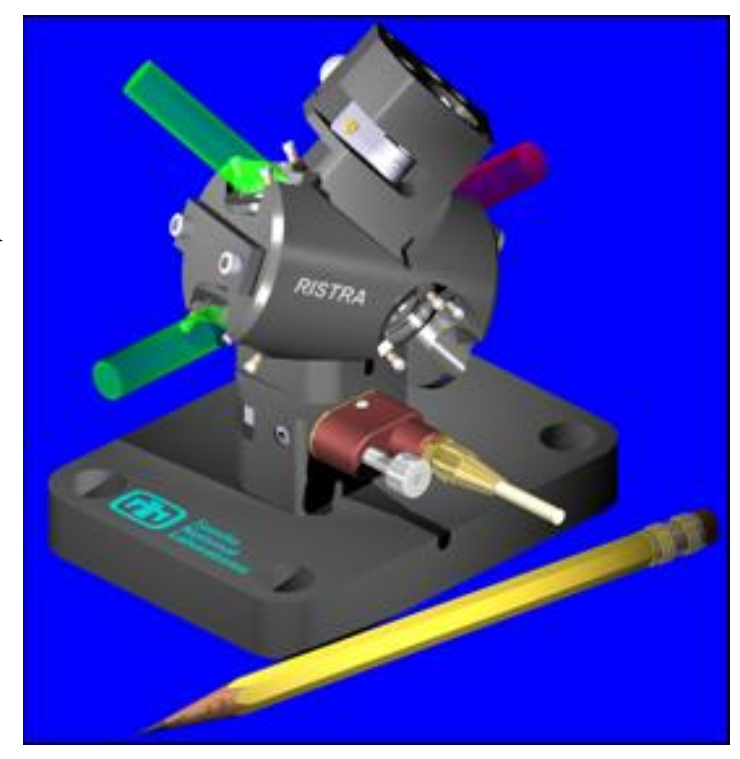

RISTRA OPO Module 


\section{Solid-State Nd:YAG Pump laser}

- For future space applications, an all solid-state, diode pumped Nd:YAG pump laser has been developed in collaboration with Fibertek, Inc.

- The pump laser is an upgrade of 300 mJ/pulse $\mathrm{Nd}$ :YAG laser developed under NASA funded ATIP program

- Two amplifiers have been added to the NASA ATIP laser to achieve up to $1.2 \mathrm{~J} /$ pulse 


\section{Nd:YAG Pump Laser \\ -Summary of Technical Approach-}

\section{An all solid-state diode-pumped laser transmitter featuring:}

- Injection seeded ring laser

- Diode-pumped zigzag slab amplifiers

- Advanced E-O phase modulator material

- Alignment insensitive / boresight stable $1.0 \mu \mathrm{m}$ cavity and optical bench

- Conduction cooled

- Space-qualifiable component designs
Improves emission brightness $\left(\mathrm{M}^{2}\right)$

Robust and efficient design for use in space

Allows high frequency cavity modulation for improved stability injection seeding

Stable and reliable operation over environment

Eliminates circulating liquids w/in cavity

Establishes a path to a space-based mission 


\section{Single Frequency Laser Ring Laser Design}

\section{Optical Schematic}

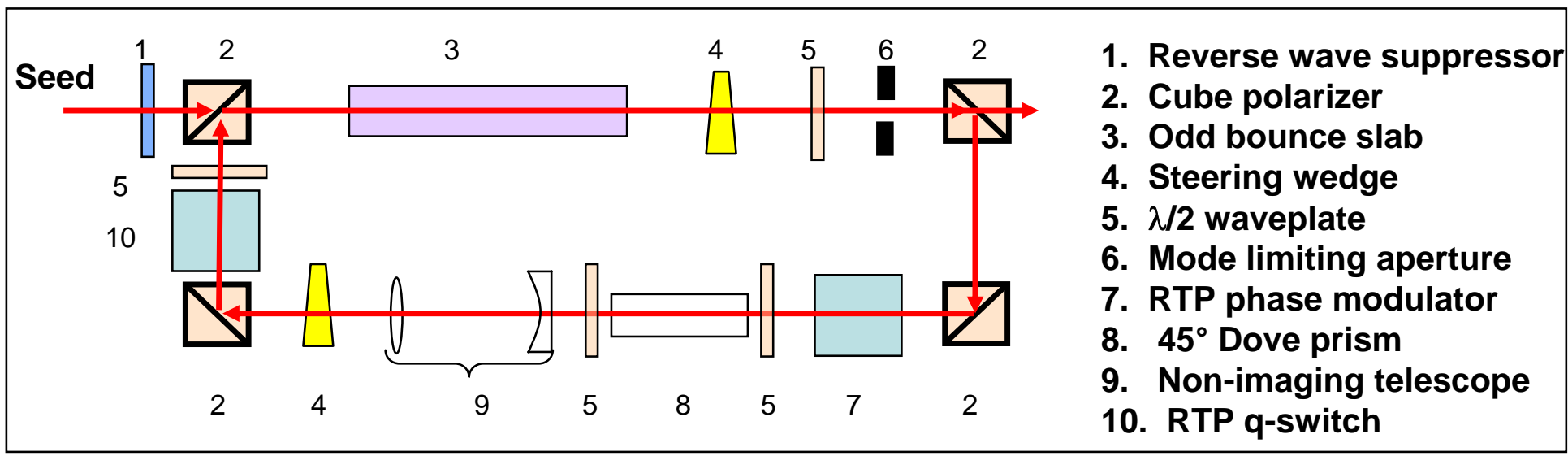

\section{Final Zerodur Optical Bench $(12 \mathrm{~cm} \times 32 \mathrm{~cm})$}

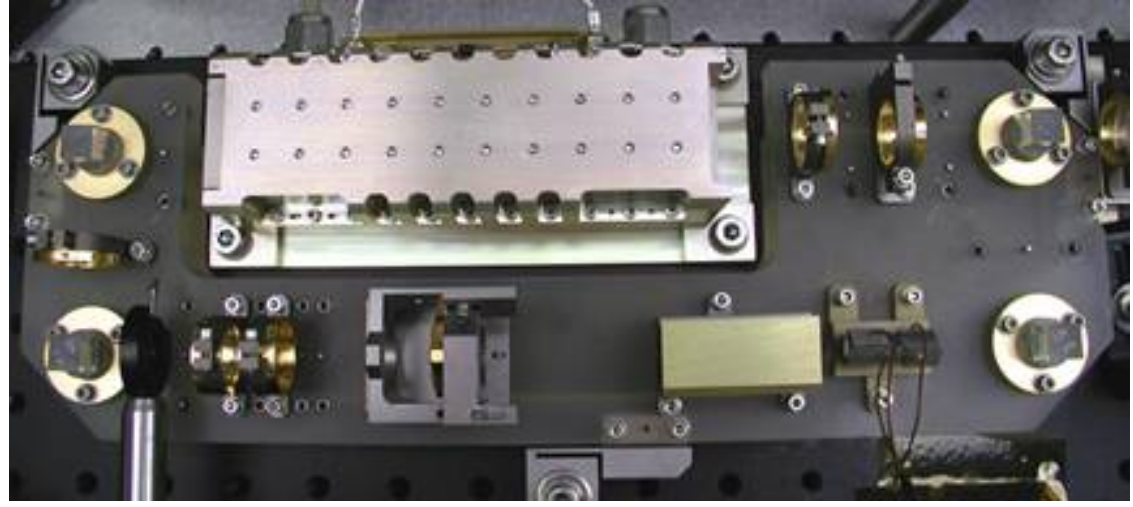

\section{Design Features}

- Near stable operation allows trading beam quality against output energy by appropriate choice of mode limiting aperture

$$
\begin{aligned}
& -30 \mathrm{~mJ} \mathrm{TEM}_{00}, \mathrm{M}^{2}=1.2 \text { at } 50 \mathrm{~Hz} \\
& -30 \mathrm{~mJ} \mathrm{TEM}_{00}, \mathrm{M}^{2}=1.3 \text { at } 100 \mathrm{~Hz} \\
& -50 \mathrm{~mJ} \text { square supergaussian, } \mathrm{M}^{2}=1.4 \\
& \text { at } 50 \mathrm{~Hz}
\end{aligned}
$$

- Injection seeding using an RTP phase modulator provides reduced sensitivity to high frequency vibration

- PZT stabilization of cavity length reduces sensitivities to thermal fluctuations

- Zerodur optical bench results in high alignment and boresight stability 


\section{Amplifier Design Configuration}

3 Bounces-Rectangular Shape-2 sided pumping in the TIR axis,

2 sided conduction cooling, Pump faces uncoated ( $10 \%$ loss)

\section{2-Sided Pumped \& Cooled Amplifier}

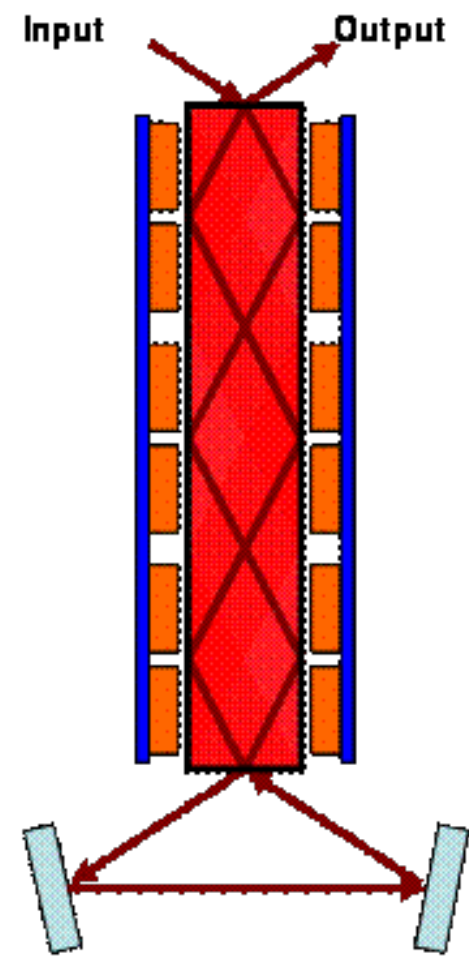

First Stage

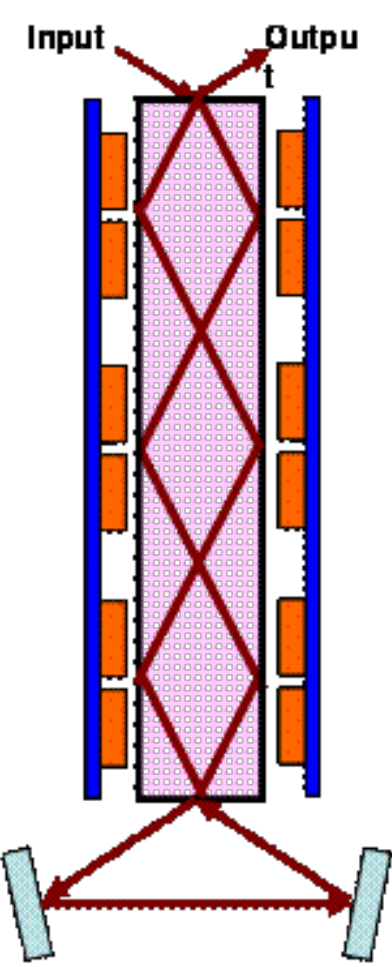

Second Stage

\section{Prototype Two-Sided Pumped and Cooled Head Design}

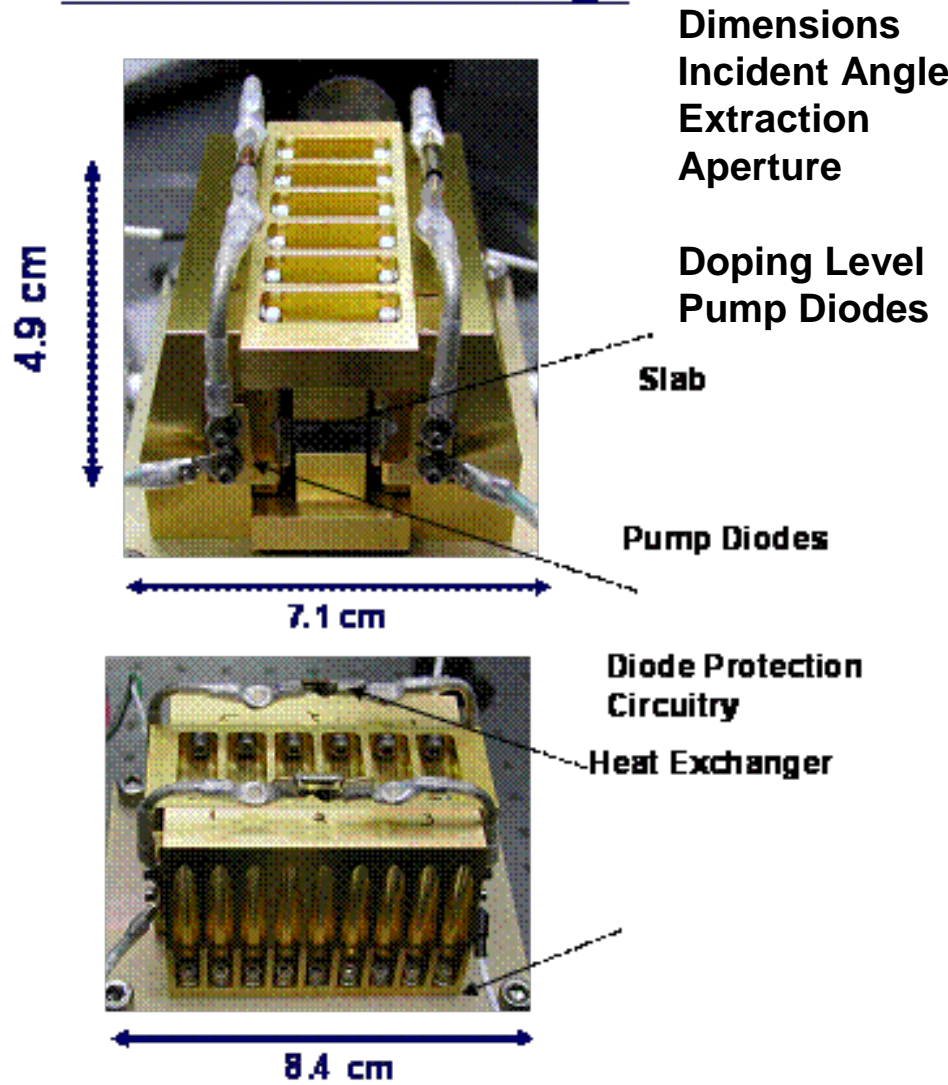

$6.8 \times 13.0 \times 75.3 \mathrm{~mm} 3$ Near Brewster (57\%) $100 \%$ at full aperture $11.5 \times 6.8 \mathrm{~mm} 2$ (internal) $7.1 \times 6.8 \mathrm{~mm} 2$ (external) $0.5 \pm 0.1 \% \mathrm{Nd} 3+$ 192 ea. 50 watt QCW bars (12 ea. 16 bar arrays) 


\section{Amplifier Upgrade 2-Sided Pumped \& Cooled Amplifier}

\section{Dual Stage Amplifier Modeling}

Model is based on Franz-Nodvic result for a amplifying a square (in time) pulse

Model includes all key parameters explicitly

- Number of pump diodes (192)

- Peak diode power (75 W)

- Diode pulse width

- Input oscillator pulse energy (60 mJ)

- Input beam diameter

- Gain path length in amp

- Slab volume

Accounts for reduced gain for second pass

$1 \mathrm{~J}$ per pulse output is predicted for $210 \mu \mathrm{s}$ diode pump pulses

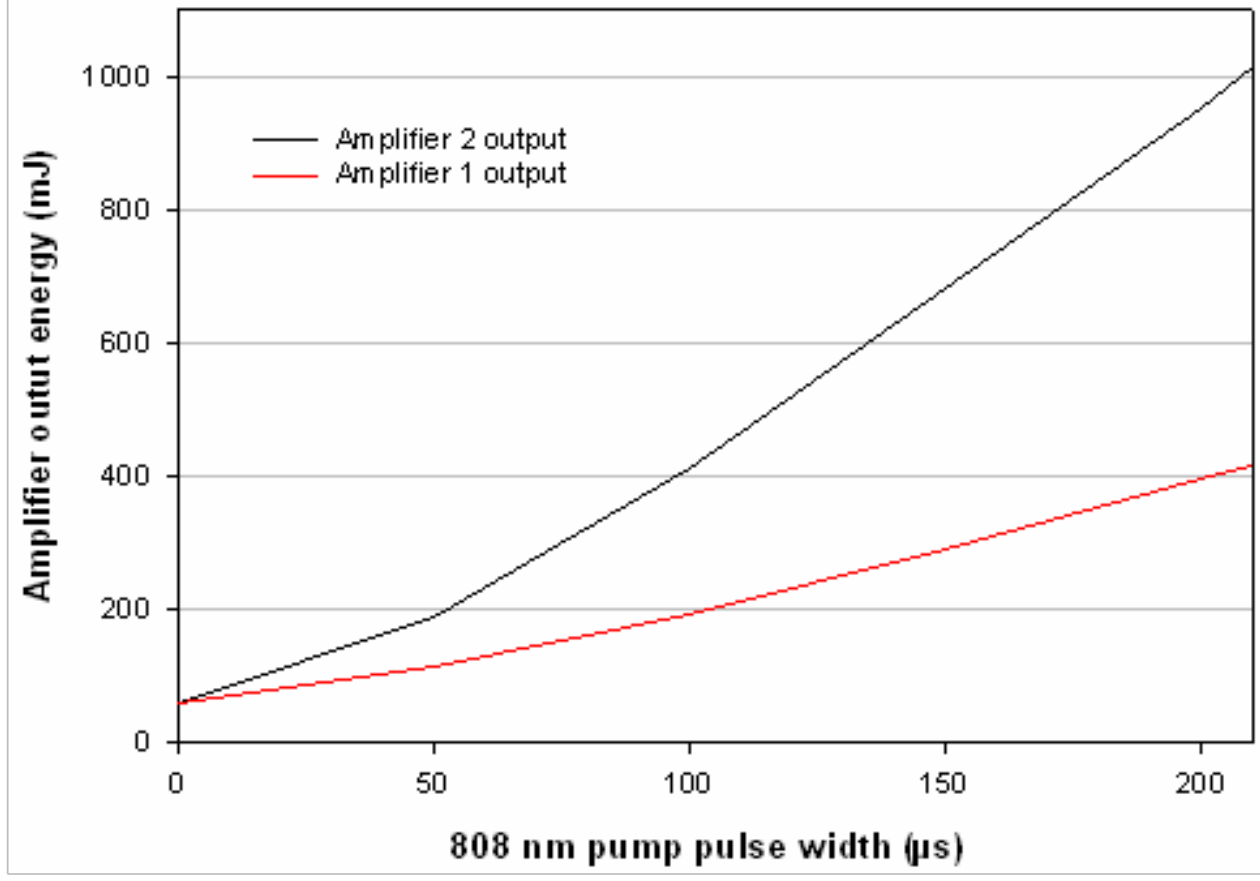

Modeled output of dual 2-sided pumped and cooled amplifiers for $60 \mathrm{~mJ}$ input to first stage

Dual 2-sided pumped amplifiers meet the requirements of most space-based direct detection wind lidars designs 


\section{Pump Laser Performance}

- The laser is now operational at $50 \mathrm{~Hz}$ PRF with maximum pulsewidths around 22 ns

- The output beam profile is rectangular super gaussian

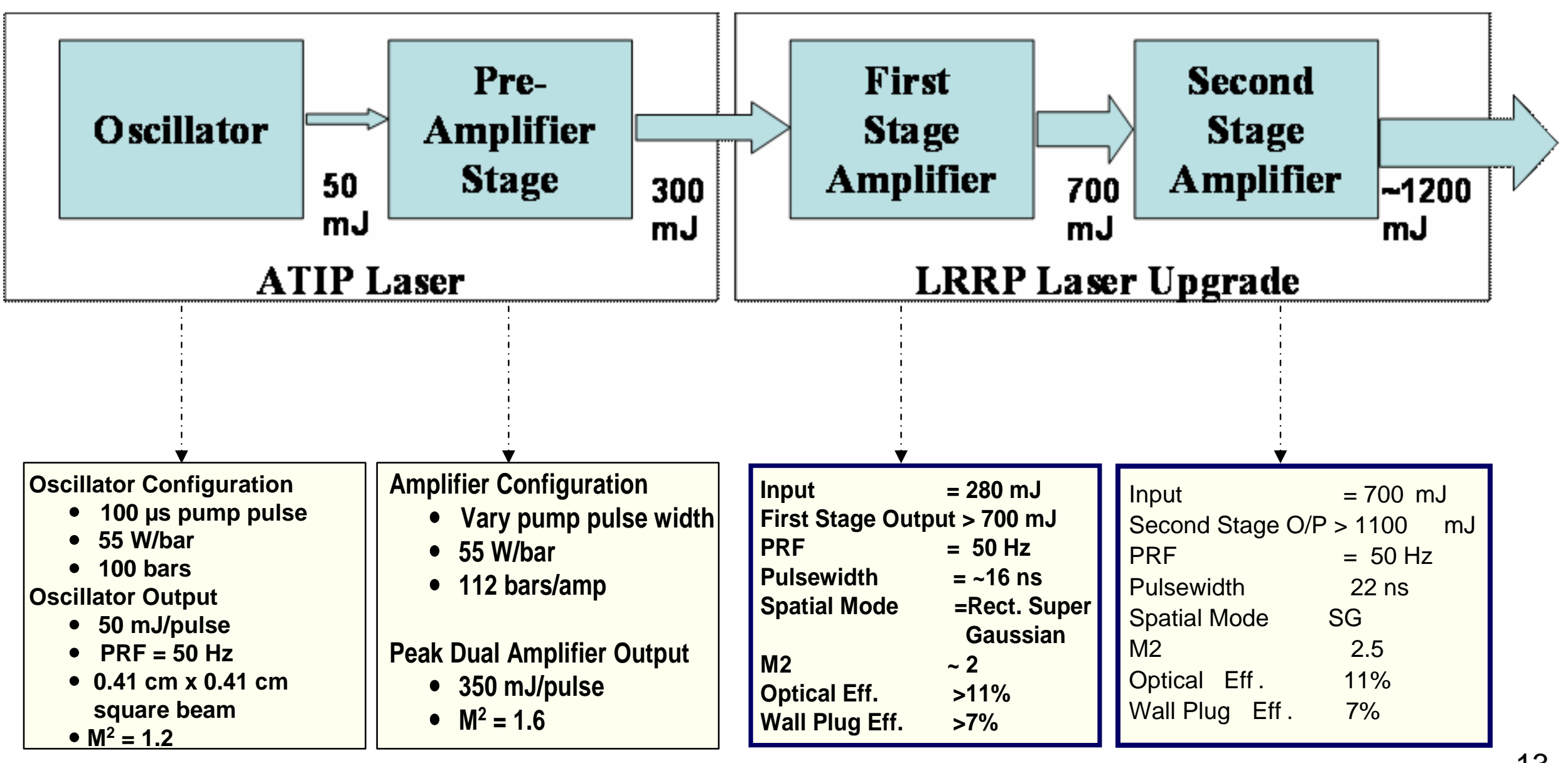




\section{Nd:YAG Pump Laser}

- Typical Output Characteristics -

\begin{tabular}{|c|c|c|c|}
\hline Parameter & Specification & Goal & Design/Performance \\
\hline $\begin{array}{c}\text { Pulse Energy } \\
(\mathrm{mJ})\end{array}$ & 900 & 1200 & 1040 \\
\hline $\mathrm{M}^{2}$ & $\mathrm{NA}$ & 2 & 2.5 \\
\hline $\begin{array}{c}\text { Laser head } \\
\text { package }\end{array}$ & Single breadboard & $\mathrm{NA}$ & $\begin{array}{c}\text { Single breadboard in } \\
\text { custom enclosure }\end{array}$ \\
\hline Cooling & $\begin{array}{c}\text { Conductive to } \\
\text { diodes and slabs }\end{array}$ & $\mathrm{NA}$ & $\begin{array}{c}\text { Conductive to diodes and } \\
\text { slabs }\end{array}$ \\
\hline Seeding & Ramp \& fire & NA & Ramp \& fire \\
\hline Electronics & $\begin{array}{c}\text { Separate custom } \\
\text { module }\end{array}$ & NA & Separate custom module \\
\hline
\end{tabular}

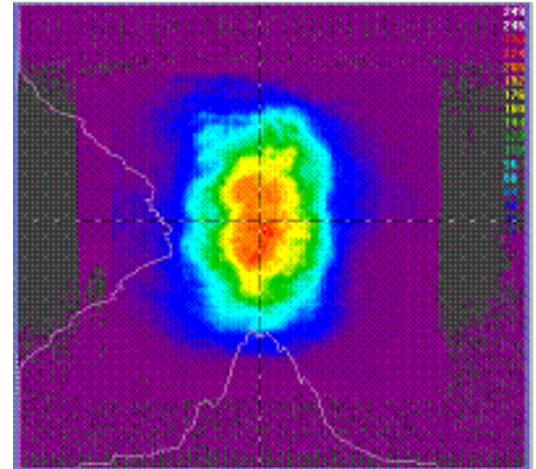

Near field beam profile of final amplifier output

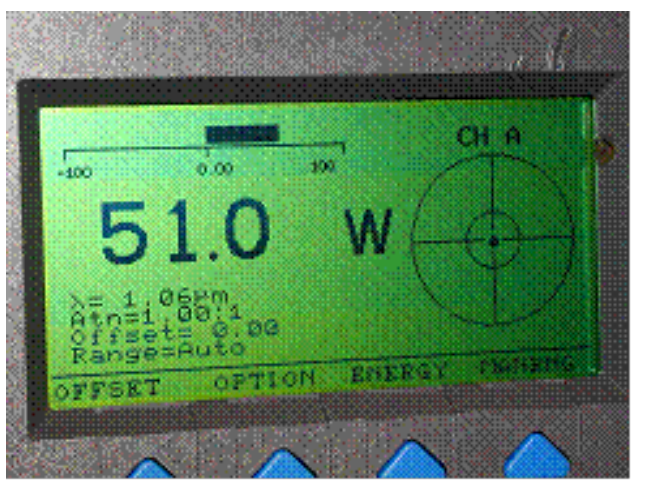

Average power at $50 \mathrm{~Hz}$ of $51.0 \mathrm{~W}$ (1020 mJ/pulse) 


\section{Temporal Characteristics}

Oscillator Only: 16.5 ns

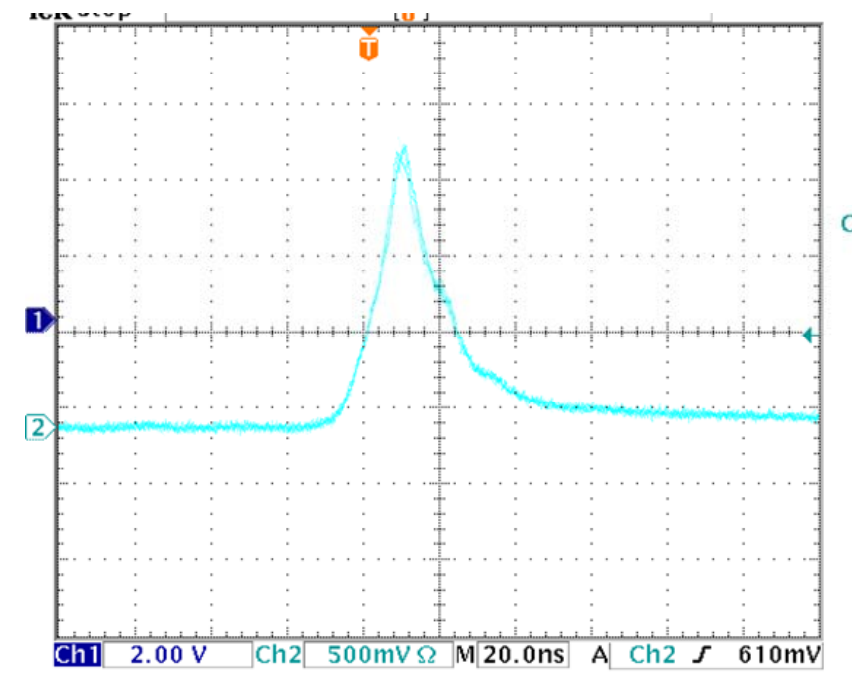

Oscillator + Amp 1 + Amp 2 : 20.9 ns
Oscillator + Preamp 1 + Preamp $2: 19.5$ ns

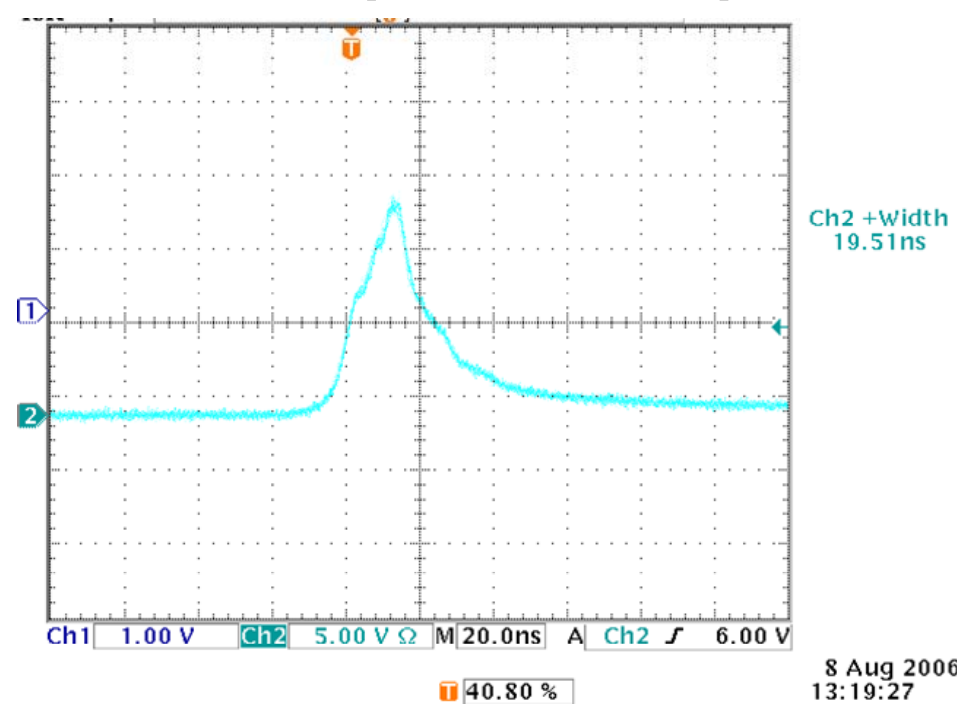

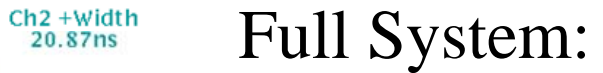

Pulsewidth $~ 22$ ns 


\section{Full System Results Beam Quality}

$50 \mathrm{~Hz}$, Full Power Beam Quality Measurements

$$
M_{x}{ }^{2}=2.5, M_{y}{ }^{2}=2.5 \text {, }
$$

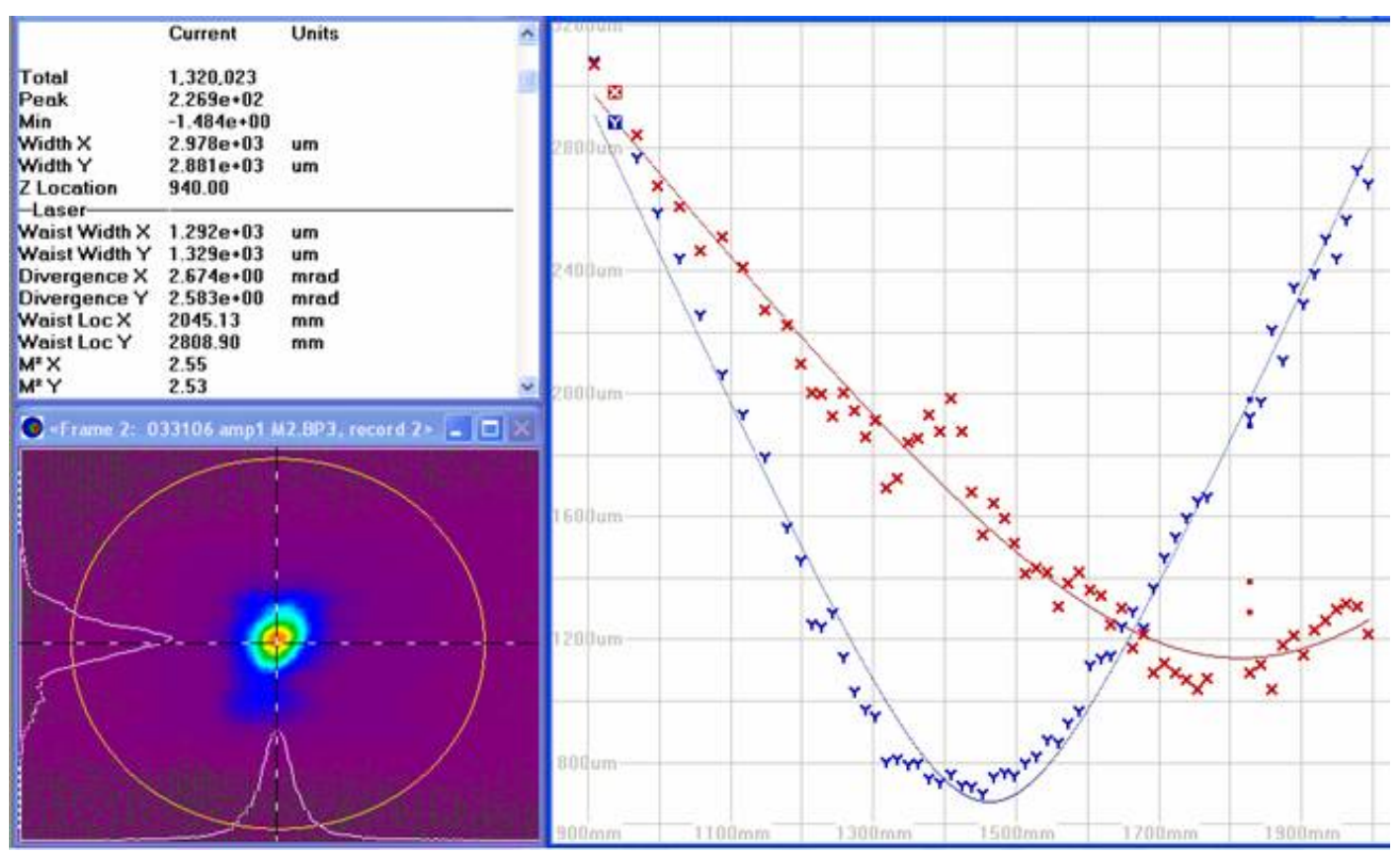

$M^{2}$ data 


\section{Full Nd:YAG Laser Unit}

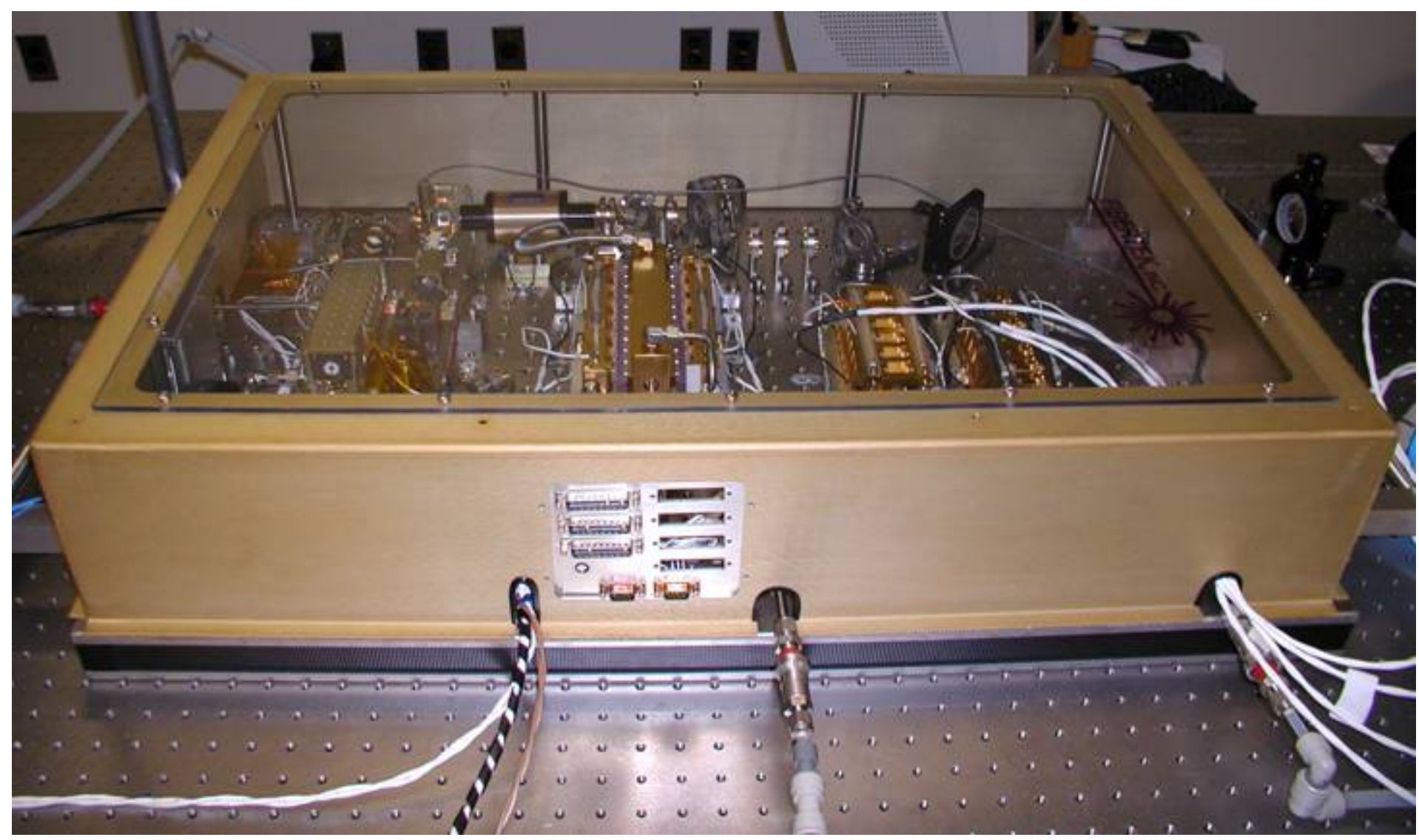

- The dimensions of this laser unit, including a SHG module, is 34” x 22" x 8"

- With latest diode bars and modified opto-mechanical components, the above package can be reduced to less than a quarter of its size 


\section{Final System \\ Control and Power Electronics}

Custom power supplies and control electronics for the upgrade have been built

- Control electronics consists of two 19"rack mountable boxes

- All power supplies are contained in two 19" rack mountable power supply modules

- Each amplifier can be individual set between high power and low power operation to allow the user to achieve a wide range of output powers at $50 \mathrm{~Hz}$

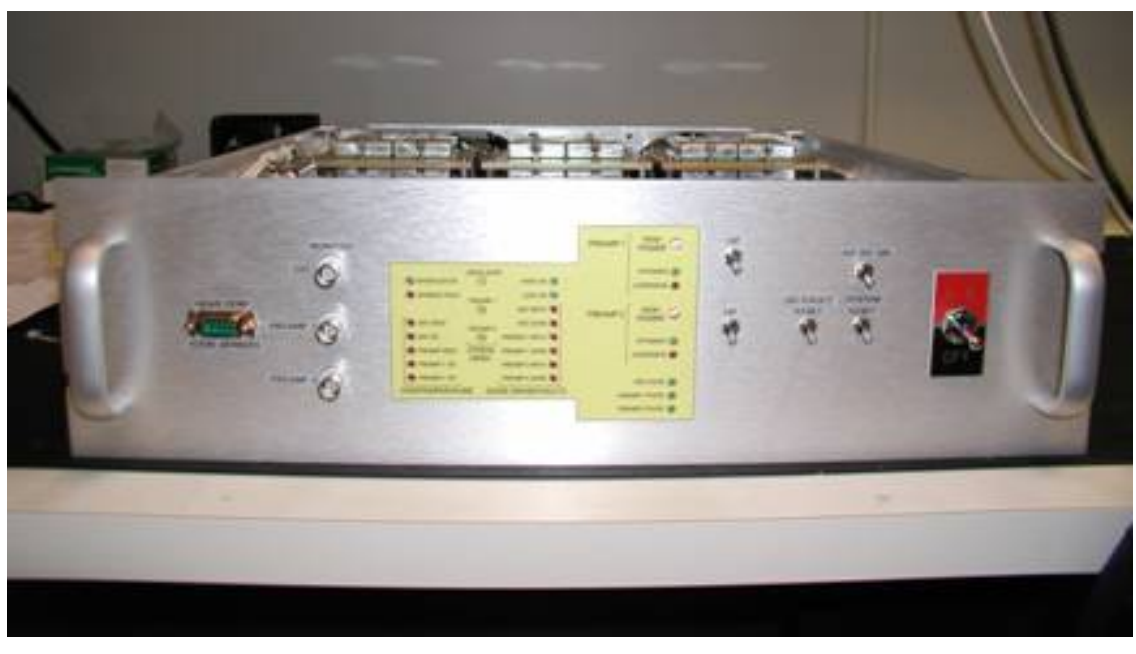

Single Power Supply Module

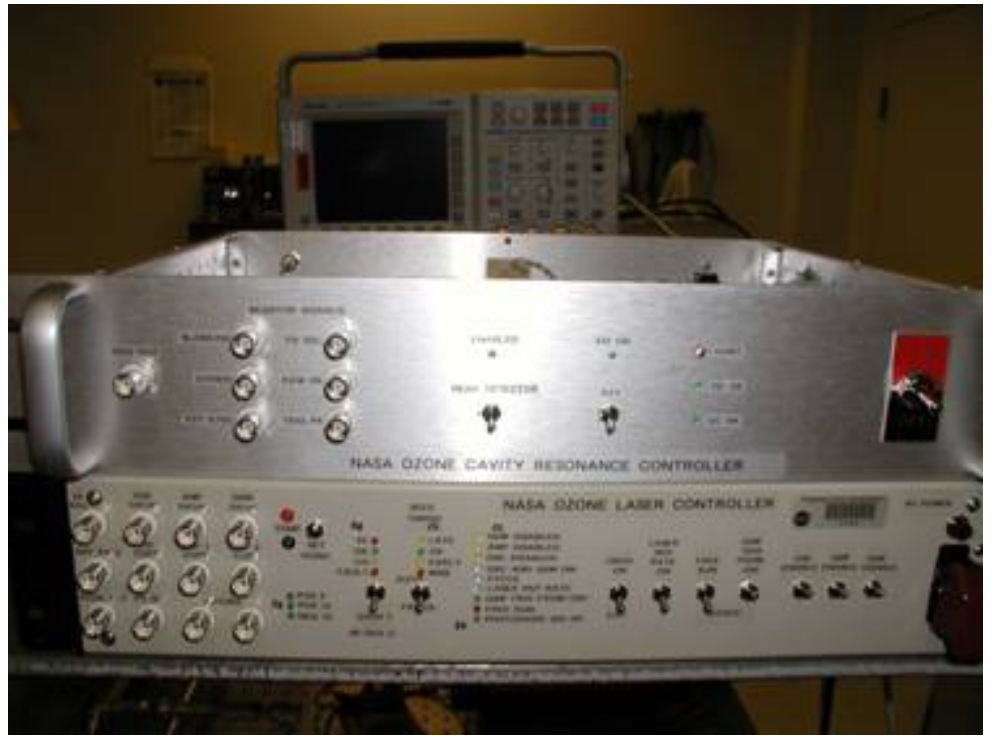

Control electronics 


\section{$320 \mathrm{~nm}$ UV generation}

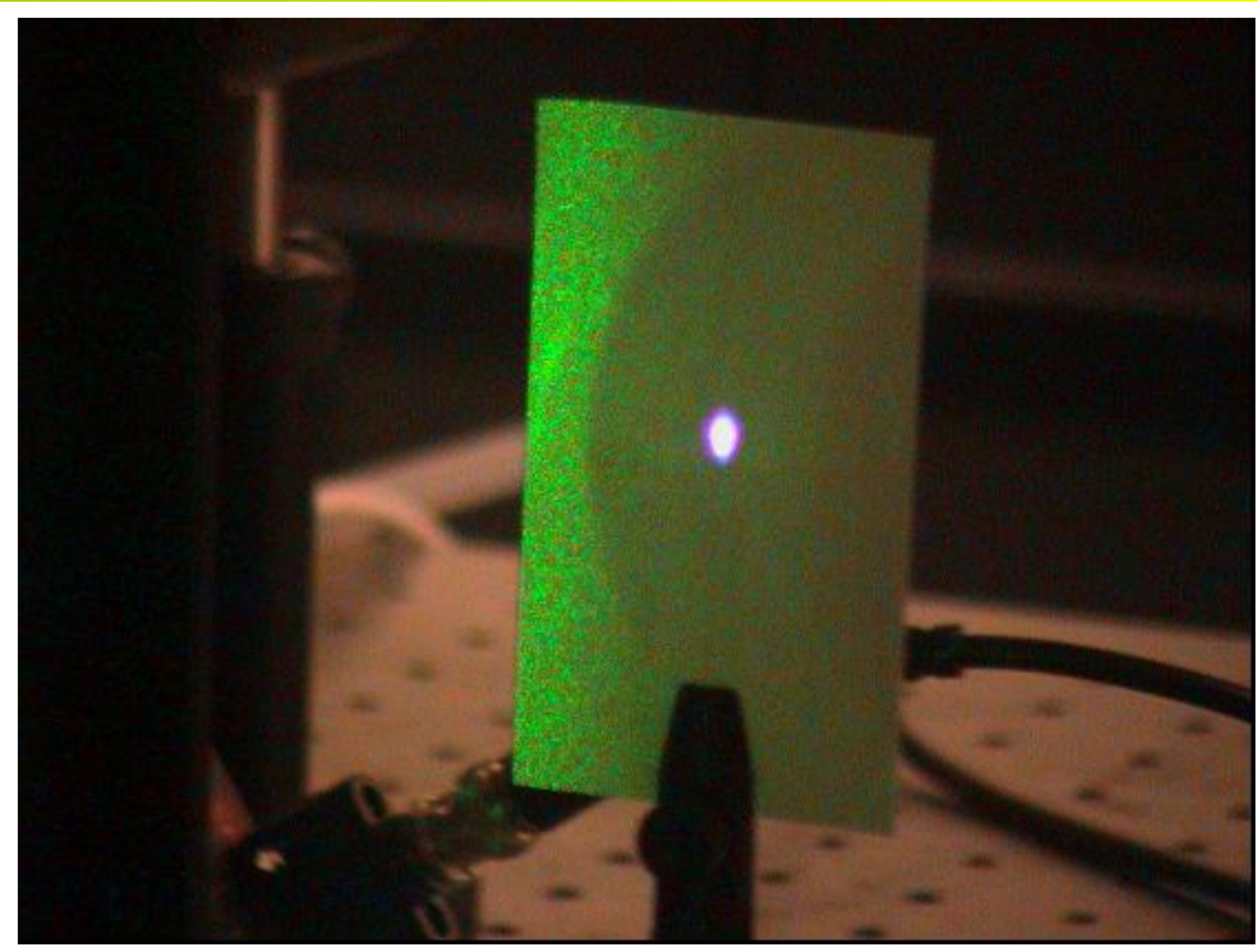

- Currently, we are generating a few mJ with limited pump energy of $280 \mathrm{~mJ} /$ pulse

- The elliptical beam allows reduced overlap inside the nonlinear crystal of RISTRA module hence reduces the conversion efficiency 


\section{Spatial fluence profile \& RISTRA}

- RISTRA OPO requires round, top-hat spatial pump profile -

\section{Flat pump profiles have facilitated high pump depletion \&hence high OPO conversion efficiency}

Results Using refined Flash Lamp pump laser

OPO signal near-field spatial fluence profile, Fresnel

Number $>450$

Self-seeded oscillation in two-crystal RISTRA $\sim 85 \%$ pump depletion
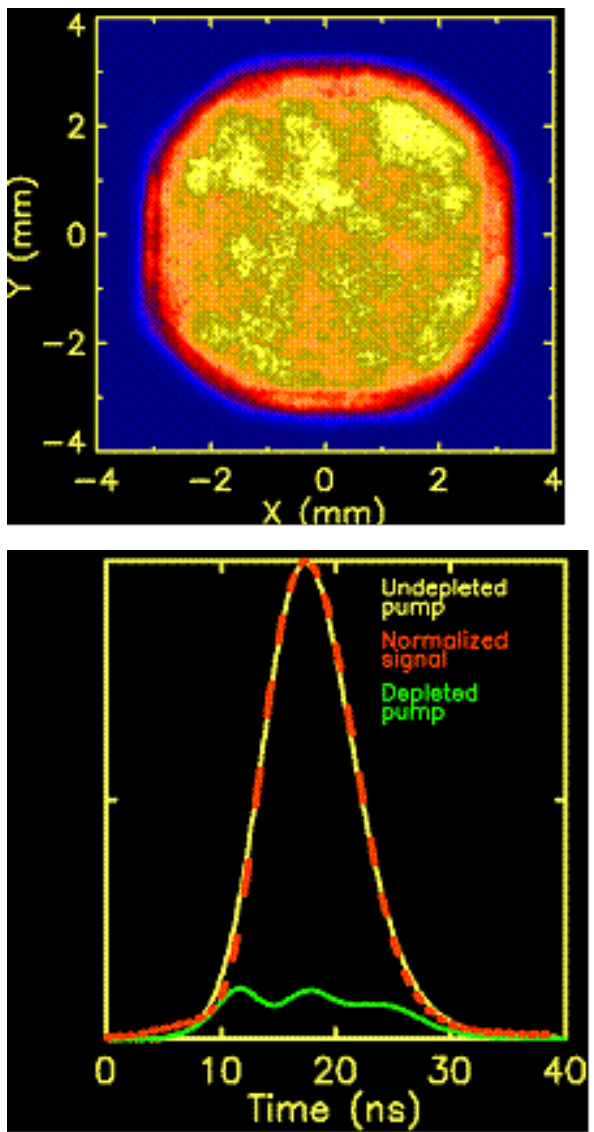

Results Using Diode pumped Nd:YAG laser

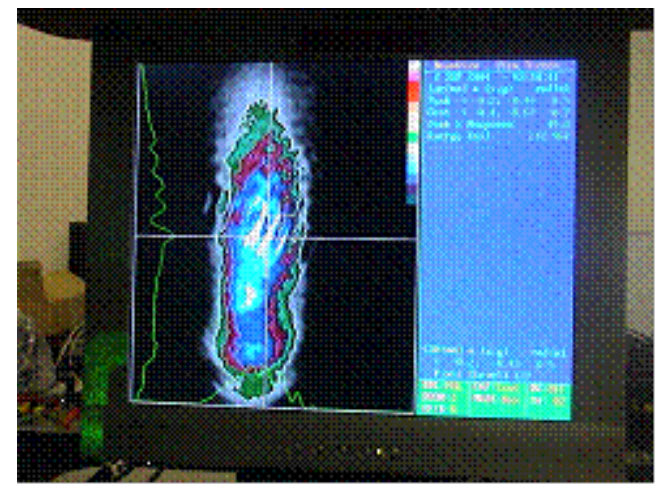

Pump Beam at the Big OPO

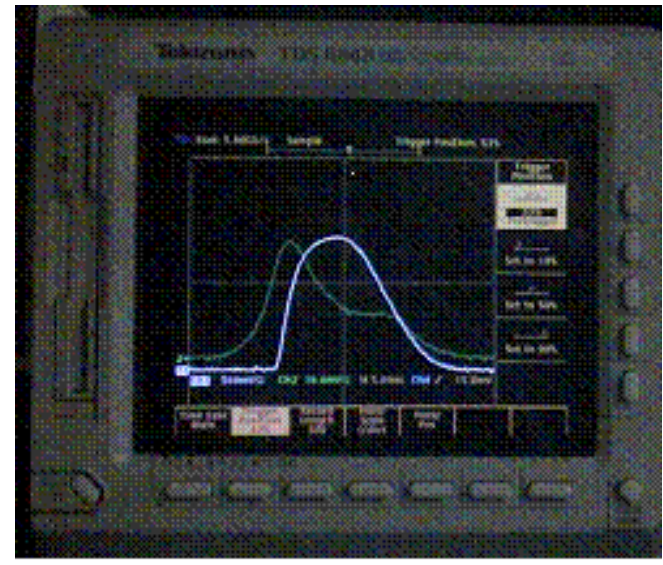

Reduced Pump Depletion 


\section{On-Going Work}

- Improve the Beam Quality of the Diode Pumped Nd:YAG Laser

- The goal is to achieve a Round, Top Hat spatial fluence profile with wavefront aberration less than 0.5
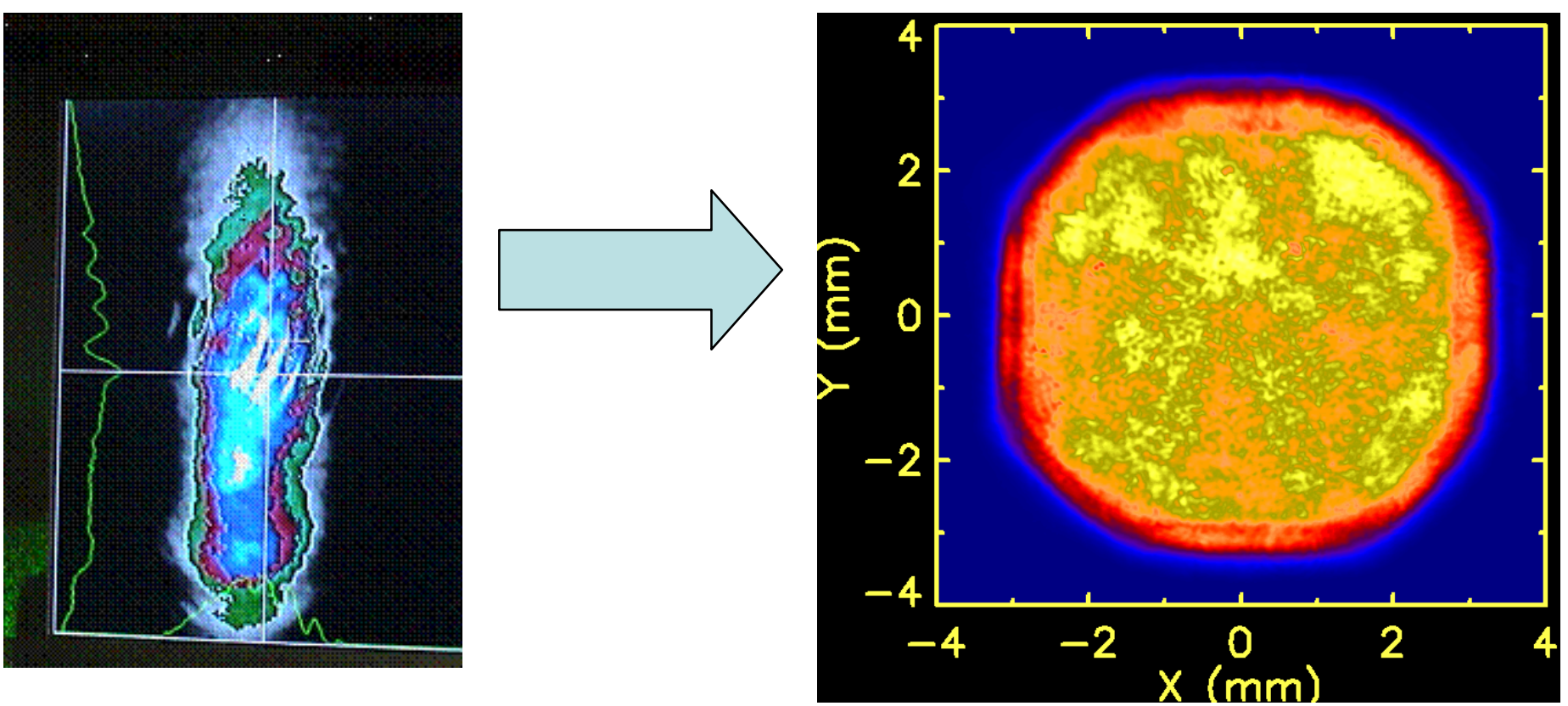

- Refinements to the ring oscillator cavity, pre amplifiers and amplifiers of the diode-pumped Nd:YAG laser to improve beam quality and reduce pulsewidth is nearing completion 


\section{Summary and Conclusions}

- A high energy, single mode, all solid-state Nd:YAG laser primarily for pumping an UV converter is developed

- Greater than $1 \mathrm{~J} /$ pulse at $50 \mathrm{HZ}$ PRF and pulsewidths around 22 ns have been demonstrated

- Higher energy, greater efficiency may be possible

- Refinements are known and practical to implement

- Technology Demonstration of a highly efficient, high-pulse-energy, single mode UV wavelength generation using flash lamp pumped laser has been achieved

- Greater than 90\% pump depletion is observed

- $190 \mathrm{~mJ}$ extra-cavity SFG; IR to UV efficiency > 21\% (> 27\% for $1 \mathrm{~mJ}$ seed)

- $160 \mathrm{~mJ}$ intra-cavity SFG; IR to UV efficiency up to $24 \%$

- Fluence $\leq 1 \mathrm{~J} / \mathrm{cm}^{2}$ for most beams

- The pump beam quality of the Nd:YAG pump laser is being refined to match or exceed the above UV converter results

- Currently the Nd:YAG pump laser development is a technology demonstration

- System can be engineered for compact packaging 